Article

\title{
Moment Bound of Solution to a Class of Conformable Time-Fractional Stochastic Equation
}

\author{
McSylvester Ejighikeme Omaba ${ }^{1}$ and Eze R. Nwaeze ${ }^{2, *(D)}$ \\ 1 Department of Mathematics, College of Science, University of Hafr Al Batin, P.O. Box 1803, Hafr Al Batin 31991, \\ Saudi Arabia; mcomaba@uhb.edu.sa \\ 2 Department of Mathematics, Tuskegee University, Tuskegee, AL 36088, USA \\ * Correspondence: enwaeze@tuskegee.edu
}

Received: 29 March 2019; Accepted: 7 April 2019; Published: 9 April 2019

\begin{abstract}
We study a class of conformable time-fractional stochastic equation $T_{\alpha, t}^{a} u(x, t)=\sigma(u(x, t)) \dot{W}_{t}$, $x \in \mathbb{R}, t \in[a, T], T<\infty, 0<\alpha<1$. The initial condition $u(x, 0)=u_{0}(x), x \in \mathbb{R}$ is a non-random function assumed to be non-negative and bounded, $T_{\alpha, t}^{a}$ is a conformable time-fractional derivative, $\sigma: \mathbb{R} \rightarrow \mathbb{R}$ is Lipschitz continuous and $\dot{W}_{t}$ a generalized derivative of Wiener process. Some precise condition for the existence and uniqueness of a solution of the class of equation is given and we also give an upper bound estimate on the growth moment of the solution. Unlike the growth moment of stochastic fractional heat equation with Riemann-Liouville or Caputo-Dzhrbashyan fractional derivative which grows in time like $t^{c_{1}} \exp \left(c_{2} t\right), c_{1}, c_{2}>0$; our result also shows that the energy of the solution (the second moment) grows exponentially in time for $t \in[a, T], T<\infty$ but with at most $c_{1} \exp \left(c_{2}(t-a)^{2 \alpha-1}\right)$ for some constants $c_{1}$, and $c_{2}$.
\end{abstract}

Keywords: conformable time-fractional derivative; energy moment growth bounds; Gaussian noise; generalized derivative; stochastic solution

\section{Introduction}

The use of fractional derivative, which is a generalization of derivative to any arbitrary order, has received a tremendous attention due to its physical and modelling applications in Science, Engineering and Mathematics, see [1] and the references. There are various definitions and generalizations of the fractional derivative, not limited to the Riemann-Liouville fractional derivative and Caputo-Dzhrbashyan fractional derivative, with their respective limitations, see [2]. One of the limitations of the above two fractional derivatives, is that they do not satisfy the classical chain rule; hence, the need for a better definition of fractional derivative. In [3], Khalil and his co-authors, introduced a better and a new well-behaved definition of a fractional derivative known as the conformable fractional derivative, satisfying the usual chain rule, the Rolle's and the mean value theorem, conformable integration by parts, fractional power series expansion and the conformal fractional derivative of the real function is zero, etc.; which has given a new research direction. Thus conformable fractional derivative is a natural extension of the classical derivative (since it can be expressed as a first derivative multiplied by a fractional factor or power) and has many advantages over other fractional derivatives as enumerated above.

Conformable fractional derivatives are applied in certain classes of conformable differentiable linear systems subject to impulsive effects and establish quantitative behaviour of the nontrivial solutions (stability, disconjugacy, etc.), see [4,5]; and used to develop the Swartzendruber model for description of 
non-Darcian flow in porous media [5-7]. Conformable derivatives are also used to solve approximate long water wave equation with conformable fractional derivative and conformable fractional differential equations via radial basis functions collocation method [8,9]. Though there has been a significant contribution in the study of class of stochastic heat equations with Riemann-Liouville (R-L) and Caputo-Dzhrbashyan (C-D) fractional derivatives [10-17], but not much has been done in the study of stochastic Cauchy equation with conformable fractional derivative, see [2], see also [18] for a recent study on conformable fractional differential equations. The challenge in studying stochastic Cauchy equation with conformable fractional derivative is that there is no singular kernel of the form $(t-s)^{-\alpha}$ generated for $0<\alpha<1$ which reflect the nonlocality and the memory in the fractional operator as in the case of $\mathrm{R}-\mathrm{L}$ and $\mathrm{C}-\mathrm{D}$ fractional derivatives. Thus, despite the fact that conformable fractional derivative combines the best characteristics of known fractional derivatives, seems more appropriate to describe the behaviour of classical viscoelastic models under interval uncertainty, see [19] and gives models that agree and are consistent with experimental data, see [20], it does not possess or satisfy a semigroup property unlike the R-L and C-D fractional operators that have well-behaved semigroup properties.

We are motivated by the fact that the conformable fractional derivative can be used to solve fractional differential equation more easily, see [18,21], and therefore consider the following class of conformable fractional stochastic equation

$$
T_{\alpha, t}^{a} u(x, t)=\sigma(u(x, t)) \dot{W}_{t}, \quad x \in \mathbb{R}, 0<a<t \leq T<\infty, 0<\alpha<1,
$$

with an initial condition $u(x, 0)=u_{0}(x)$; where $T_{\alpha, t}^{a}$ is a conformable fractional derivative, $\sigma: \mathbb{R} \rightarrow \mathbb{R}$ is a Lipschitz continuous function and $\dot{W}_{t}$ is a generalized derivative of Wiener process (Gaussian white noise). The existence and uniqueness result is given and we also give the moment growth bound estimate on the solution of the above equation. Similar models have been considered for Caputo derivatives [22-24] where existence and uniqueness results were studied. To the best of our knowledge, we are the first to consider this model for the conformable fractional derivative. We prove the existence and uniqueness result and also give the moment growth bound estimate on the solution of the above equation.

The paper is outlined as follows. In Section 2, we give a brief overview of basic concepts used in this paper. The problem formulation, the main results, their proofs are given in Section 3, and Section 4 contains some theoretical examples to illustrate our result. We end with a short conclusion in Section 5.

\section{Preliminaries}

We give the definition of conformable fractional derivative, see $[1-3,18,25,26]$ and their references for details on conformable fractional derivatives. Let $\mathcal{D}:=[a, \infty) \times \mathbb{R}$.

Definition 1. The conformable time-fractional derivative starting from a of a function $u: \mathcal{D} \rightarrow \mathbb{R}$ of order $\alpha$ is

$$
T_{\alpha, t}^{a} u(x, t)=\lim _{\epsilon \rightarrow 0} \frac{u\left(x, t+\epsilon(t-a)^{1-\alpha}\right)-u(x, t)}{\epsilon} .
$$

If $T_{\alpha, t}^{a} u(x, t)$ exists on $(a, b)$, then

$$
T_{\alpha, t}^{a} u(x, a)=\lim _{t \rightarrow a} T_{\alpha, t}^{a} u(x, t)
$$

and if $u_{t}(x, t)$ exists then

$$
T_{\alpha, t}^{a} u(x, t)=(t-a)^{1-\alpha} u_{t}(x, t) .
$$


Definition 2. The fractional integral starting from a of a function $u: \mathcal{D} \rightarrow \mathbb{R}$ of order $\alpha$ is

$$
T_{\alpha, t}^{a} u(x, t)=\int_{a}^{t}(s-a)^{\alpha-1} u(x, s) d s .
$$

We first give a generalized derivative for a deterministic function $w(t)$.

Definition 3. Given that $g(t)$ is any smooth and compactly supported function, then we define the generalized derivative $\dot{w}(t)$ of $w(t)$ (not necessarily differentiable) as

$$
\int_{0}^{\infty} g(t) \dot{w}(t) d t=-\int_{0}^{\infty} \dot{g}(t) w(t) d t
$$

Similarly, the generalized derivative $\dot{W}_{t}$ of Wiener process with a smooth function $g(t)$ as follows:

$$
\int_{0}^{t} g(s) \dot{W}_{s} d s=g(t) W_{t}-\int_{0}^{t} \dot{g}(s) W_{s} d s
$$

We give the gamma function as follows

$$
\Gamma(z)=\gamma(z, x)+\Gamma(z, x)
$$

where $\gamma(z, x)$ is an incomplete gamma function given by

$$
\gamma(z, x)=\int_{0}^{x} e^{-t} t^{z-1} d t, x>0
$$

and $\Gamma(z, x)$ is the complement of the incomplete gamma function given by

$$
\Gamma(z, x)=\int_{x}^{\infty} e^{-t} t^{z-1} d t, x>0 .
$$

Next, we give some estimates (bounds) on the incomplete gamma function.

Theorem 1 ([27]). The following inequalities

$$
\exp \left(\frac{-a x}{a+1}\right) \leq \frac{a}{x^{a}} \gamma(a, x) \leq{ }_{1} F_{1}(a ; a+1 ;-x) \leq \frac{1}{a+1}\left(1+a e^{-x}\right)
$$

hold, where ${ }_{1} F_{1}(a ; a+1 ;-x)$ is a confluent hypergeometric (Kummer) function.

Also, for $0<a \leq 1$,

$$
\frac{1-e^{-x}}{x} \leq \frac{a}{x^{a}} \gamma(a, x)
$$

\section{Main Results}

Assume the following condition on $\sigma$; that is, $\sigma$ is globally Lipschitz:

Condition 1. There exist a finite positive constant, $\operatorname{Lip}_{\sigma}$ such that for all $x, y \in \mathbb{R}$, we have

$$
|\sigma(x)-\sigma(y)| \leq \operatorname{Lip}_{\sigma}|x-y|
$$

with $\sigma(0)=0$ for convenience. 
Also, the assumption on $u$ :

Condition 2. The random solution $u: \mathcal{D} \rightarrow \mathbb{R}$ is $L^{2}$-continuous (or continuous in probability).

Define the following $L^{2}(\mathbb{P})$ norm

$$
\|u\|_{2, \alpha, \beta}:=\left\{\sup _{a \leq t \leq T} \sup _{x \in \mathbb{R}} e^{-\frac{\beta}{\alpha}(t-a)^{\alpha}} \mathbb{E}|u(x, t)|^{2}\right\}^{1 / 2} .
$$

Following similar idea in [18], we give the following results:

Lemma 1. Given that Condition 2 holds, then a function $u$ in $L^{2}(\mathbb{P})$ is a solution of Equation (1) if and only if it is a solution of the integral equation

$$
u(x, t)=u_{0}(x)+I_{\alpha, t}^{a}\left[\sigma(u(x, t)) \dot{W}_{t}\right]
$$

Thus, the solution to Equation (1) is given as follows

$$
\begin{aligned}
u(x, t) & =u_{0}(x)+\int_{a}^{t}(s-a)^{\alpha-1} \sigma(u(x, s)) \dot{W}_{s} d s \\
& =u_{0}(x)+\int_{a}^{t}(s-a)^{\alpha-1} \sigma(u(x, s)) d W_{s}
\end{aligned}
$$

Theorem 2. Suppose $\mathcal{C}_{\alpha, \beta, T}<\frac{1}{\operatorname{Lip}_{\sigma}^{2}}$ for positive constant $\operatorname{Lip}_{\sigma}$ together with both Conditions 1 and 2 . Then there exists solution $u$ that is unique up to modification, with

$$
\mathcal{C}_{\alpha, \beta, T}:=\frac{(T-a)^{2 \alpha-1}}{2 \alpha(2 \alpha-1)}\left(1+(2 \alpha-1) e^{\frac{\beta}{\alpha}(T-a)}\right) .
$$

We start by defining the operator

$$
\mathcal{A} u(x, t)=u_{0}(x)+\int_{a}^{t}(s-a)^{\alpha-1} \sigma(u(x, s)) d W_{s},
$$

and the fixed point of the operator gives the solution of Equation (1).

The proof of the theorem is based on the following lemmas:

Lemma 2. Suppose $u$ is a predictable random solution such that $\|u\|_{2, \alpha, \beta}<\infty$ and Conditions 1 and 2 hold. Then there exists a positive constant $\mathcal{C}_{\alpha, \beta, T}$ such that

$$
\|\mathcal{A} u\|_{2, \alpha, \beta}^{2} \leq c_{1}+\mathcal{C}_{\alpha, \beta, T} \operatorname{Lip}_{\sigma}^{2}\|u\|_{2, \alpha, \beta}^{2}
$$

Proof. By the assumption that $u_{0}$ is bounded, we obtain

$$
\mathbb{E}|\mathcal{A} u(x, t)|^{2} \leq c_{1}+\operatorname{Lip}_{\sigma}^{2} \int_{a}^{t}(s-a)^{2(\alpha-1)} \mathbb{E}|u(x, s)|^{2} d s .
$$


Multiply through by $e^{-\frac{\beta}{\alpha}(t-a)^{\alpha}}$ to obtain

$$
\begin{aligned}
e^{-\frac{\beta}{\alpha}(t-a)^{\alpha}} \mathbb{E}|\mathcal{A} u(x, t)|^{2} & \leq c_{1} \mathrm{e}^{-\frac{\beta}{\alpha}(t-a)^{\alpha}}+\operatorname{Lip}_{\sigma}^{2} e^{-\frac{\beta}{\alpha}(t-a)^{\alpha}} \int_{a}^{t}(s-a)^{2(\alpha-1)} \mathrm{e}^{\frac{\beta}{\alpha}(s-a)^{\alpha}} e^{-\frac{\beta}{\alpha}(s-a)^{\alpha}} \mathbb{E}|u(x, s)|^{2} d s \\
& \leq c_{1} e^{-\frac{\beta}{\alpha}(t-a)^{\alpha}}+\operatorname{Lip}_{\sigma}^{2} e^{-\frac{\beta}{\alpha}(t-a)^{\alpha}}\|u\|_{2, \alpha, \beta}^{2} \int_{a}^{t}(s-a)^{2(\alpha-1)} e^{\frac{\beta}{\alpha}(s-a)^{\alpha}} d s \\
& \leq c_{1}+\operatorname{Lip}_{\sigma}^{2}\|u\|_{2, \alpha, \beta}^{2} \int_{a}^{t}(s-a)^{2(\alpha-1)} e^{\frac{\beta}{\alpha}(s-a)^{\alpha}} d s
\end{aligned}
$$

since $e^{-\frac{\beta}{\alpha}(t-a)^{\alpha}} \leq 1, a \leq t \leq T$, that is, $0 \leq t-a \leq T-a \Rightarrow-\frac{\beta}{\alpha}(t-a) \leq 0$.

Thus taking sup over $t \in[a, T]$ and $x \in \mathbb{R}$ and evaluating the integral we have

$$
\begin{aligned}
\|\mathcal{A} u\|_{2, \alpha, \beta}^{2} & \leq c_{1}+\operatorname{Lip}_{\sigma}^{2}\|u\|_{2, \alpha, \beta}^{2} \int_{a}^{t}(s-a)^{2(\alpha-1)} e^{\frac{\beta}{\alpha}(s-a)^{\alpha}} d s \\
& \leq c_{1}+\operatorname{Lip}_{\sigma}^{2}\|u\|_{2, \alpha, \beta}^{2} \times-\frac{\beta}{\alpha}(t-a)^{2 \alpha}\left(\frac{\beta}{\alpha}\right)^{-2 \alpha}(a-t)^{-2 \alpha}\left[\Gamma(2 \alpha-1)-\Gamma\left(2 \alpha-1, \frac{\beta}{\alpha}(a-t)\right)\right] \\
& \leq c_{1}+\operatorname{Lip}_{\sigma}^{2}\|u\|_{2, \alpha, \beta}^{2} \times-(-1)^{-2 \alpha}\left(\frac{\beta}{\alpha}\right)^{1-2 \alpha}\left[\Gamma(2 \alpha-1)-\Gamma\left(2 \alpha-1, \frac{\beta}{\alpha}(a-t)\right)\right] \\
& \leq c_{1}+\operatorname{Lip}_{\sigma}^{2}\left(\frac{\beta}{\alpha}\right)^{1-2 \alpha} \gamma\left(2 \alpha-1, \frac{\beta}{\alpha}(a-t)\right)\|u\|_{2, \alpha, \beta}^{2} .
\end{aligned}
$$

By the estimate on the incomplete gamma function in Theorem 1, we obtain

$$
\begin{aligned}
\gamma\left(2 \alpha-1, \frac{\beta}{\alpha}(a-t)\right) & \leq \frac{\left(\frac{\beta}{\alpha}\right)^{2 \alpha-1}}{2 \alpha(2 \alpha-1)}(a-t)^{2 \alpha-1}\left(1+(2 \alpha-1) e^{\frac{\beta}{\alpha}(t-a)}\right) \\
& \leq \frac{\left(\frac{\beta}{\alpha}\right)^{2 \alpha-1}}{2 \alpha(2 \alpha-1)}(t-a)^{2 \alpha-1}\left(1+(2 \alpha-1) e^{\frac{\beta}{\alpha}(t-a)}\right)
\end{aligned}
$$

and therefore, since $0<t-a<T-a$, we have

$$
\begin{aligned}
\|\mathcal{A} u\|_{2, \alpha, \beta}^{2} & \leq c_{1}+\frac{\operatorname{Lip}_{\sigma}^{2}}{2 \alpha(2 \alpha-1)}(t-a)^{2 \alpha-1}\left(1+(2 \alpha-1) e^{\frac{\beta}{\alpha}(t-a)}\right)\|u\|_{2, \alpha, \beta}^{2} \\
& \leq c_{1}+\frac{\operatorname{Lip}_{\sigma}^{2}}{2 \alpha(2 \alpha-1)}(T-a)^{2 \alpha-1}\left(1+(2 \alpha-1) e^{\frac{\beta}{\alpha}(T-a)}\right)\|u\|_{2, \alpha, \beta}^{2} .
\end{aligned}
$$

Lemma 3. Suppose $u$ and $v$ are predictable random solutions such that $\|u\|_{2, \alpha, \beta}+\|v\|_{2, \alpha, \beta}<\infty$ and Conditions 1 and 2 hold. Then there exists a positive constant $\mathcal{C}_{\alpha, \beta, T}$ such that

$$
\|\mathcal{A} u-\mathcal{A} v\|_{2, \alpha, \beta}^{2} \leq \mathcal{C}_{\alpha, \beta, T} \operatorname{Lip}_{\sigma}^{2}\|u-v\|_{2, \alpha, \beta}^{2}
$$

Remark 1. By Fixed point theorem we have $u(x, t)=\mathcal{A} u(x, t)$ and

$$
\|u\|_{2, \alpha, \beta}^{2}=\|\mathcal{A} u\|_{2, \alpha, \beta}^{2} \leq c_{1}+\mathcal{C}_{\alpha, \beta, T} \operatorname{Lip}_{\sigma}^{2}\|u\|_{2, \alpha, \beta}^{2}
$$


which follows that

$$
\|u\|_{2, \alpha, \beta}^{2}\left[1-\mathcal{C}_{\alpha, \beta, T} \operatorname{Lip}_{\sigma}^{2}\right] \leq c_{1} \Rightarrow\|u\|_{2, \alpha, \beta}<\infty \Leftrightarrow \mathcal{C}_{\alpha, \beta, T}<\frac{1}{\operatorname{Lip}_{\sigma}^{2}}
$$

Similarly,

$$
\|u-v\|_{2, \alpha, \beta}^{2}=\|\mathcal{A} u-\mathcal{A} v\|_{2, \alpha, \beta}^{2} \leq \mathcal{C}_{\alpha, \beta, T} \operatorname{Lip}_{\sigma}^{2}\|u-v\|_{2, \alpha, \beta}^{2}
$$

thus $\|u-v\|_{2, \alpha, \beta}^{2}\left[1-\mathcal{C}_{\alpha, \beta, T} \operatorname{Lip}_{\sigma}^{2}\right] \leq 0$ and therefore $\|u-v\|_{2, \alpha, \beta}<0$ if and only if $\mathcal{C}_{\alpha, \beta, T}<\frac{1}{\operatorname{Lip}_{\sigma}^{2}}$.

The existence and uniqueness result follows by Banach's contraction principle.

Next, we give the growth moment bound (upper bound estimate) on the solution:

Theorem 3. Given that Conditions 1 and 2 hold, then for $t \in[a, T], 0<T<\infty$ we have

$$
\sup _{x \in \mathbb{R}} \mathbb{E}|u(x, t)|^{2} \leq c_{1} \exp \left(c_{2}(t-a)^{2 \alpha-1}\right)
$$

for some positive constant $c_{1}$ and $c_{2}=\frac{\operatorname{Lip}_{\sigma}^{2}}{2 \alpha-1}, \alpha \neq \frac{1}{2}$.

Proof. Assume that the initial condition $u_{0}(x)$ is bounded, then by Itó isometry, we have

$$
\begin{aligned}
\mathbb{E}|u(x, t)|^{2} & \leq\left|u_{0}(x)\right|^{2}+\int_{a}^{t}(s-a)^{2(\alpha-1)} \mathbb{E}|\sigma(u(x, s))|^{2} d s \\
& \leq c_{1}+\operatorname{Lip}_{\sigma}^{2} \int_{a}^{t}(s-a)^{2(\alpha-1)} \mathbb{E}|u(x, s)|^{2} d s .
\end{aligned}
$$

Let $g(t):=\sup _{x \in \mathbb{R}} \mathbb{E}|u(x, t)|^{2}$. Then by Gronwall's inequality,

$$
\begin{aligned}
g(t) & \leq c_{1}+\operatorname{Lip}_{\sigma}^{2} \int_{a}^{t}(s-a)^{2(\alpha-1)} g(s) d s \leq c_{1} \exp \left[\operatorname{Lip}_{\sigma}^{2} \int_{a}^{t}(s-a)^{2(\alpha-1)} d s\right] \\
& =c_{1} \exp \left[\frac{\operatorname{Lip}_{\sigma}^{2}}{2 \alpha-1}(t-a)^{2 \alpha-1}\right]
\end{aligned}
$$

and the result follows.

\section{Some Examples}

Here, we give some theoretical examples.

1. Consider the following stochastic conformable fractional equation

$$
T_{\frac{2}{3}, t}^{a} u(x, t)=\sigma(u(x, t)) \dot{W}_{t}
$$

with a bounded initial function $u_{0}(x)=\frac{1}{1+e^{-\lambda x}} \lambda>0$, then the solution is given as

$$
u(x, t)=\frac{1}{1+e^{-\lambda x}}+\int_{a}^{t}(s-a)^{-1 / 2} \sigma(u(x, s)) d W_{s}
$$

and the growth bound of 


$$
\mathbb{E}|u(x, t)|^{2} \leq \sup _{x \in \mathbb{R}} \mathbb{E}|u(x, t)|^{2} \leq \exp \left(3 \operatorname{Lip}_{\sigma}^{2} \sqrt[3]{t-a}\right)
$$

The initial condition $u_{0}(x)$ can take any of the following functions $u_{0}(x)=\tan ^{-1}(x)$ or $u_{0}(x)=f_{\alpha}(x)=\frac{1}{1+x^{\alpha-1}}, \alpha>0$.

2. Consider also the conformable fractional equation

$$
T_{\frac{1}{4}, t}^{a} u(x, t)=\sigma(u(x, t)) \dot{W}_{t}
$$

with a bounded initial function $u_{0}(x)=\tan ^{-1}(x)$, then we give the solution as

$$
u(x, t)=\tan ^{-1}(x)+\int_{a}^{t}(s-a)^{-3 / 4} \sigma(u(x, s)) d W_{s}
$$

with the growth bound estimate of

$$
\mathbb{E}|u(x, t)|^{2} \leq \sup _{x \in \mathbb{R}} \mathbb{E}|u(x, t)|^{2} \leq \frac{\pi}{2} \exp \left(-\frac{2 \operatorname{Lip}_{\sigma}^{2}}{\sqrt{t-a}}\right)
$$

\section{Conclusions}

A class of conformable time-fractional stochastic equation was given, the existence and uniqueness result obtained under some precise conditions and we gave the second moment energy bound of the solution. The result showed that our solution grows exponentially in time with a precise rate of at most $c_{1} \exp \left(c_{2}(t-a)^{2 \alpha-1}\right), t \in[a, T], T<\infty$ and $0<\alpha<1$.

Author Contributions: All authors read and approved the final manuscript.

Funding: The authors did not receive any external funding.

Acknowledgments: Many thanks to the two anonymous referees for their valuable comments and suggestions.

Conflicts of Interest: The authors declare that there is no competing interests.

\section{References}

1. Zao, D.; Luo, M. General conformable fractional derivative and its physical interpretation. Calcolo 2017, 54, 903-917. [CrossRef]

2. Cenesis, Y.; Kurt, A.; Nane, E. Stochastic solutions of Conformable fractional Cauchy problems. Stat. Probab. Lett. 2017, 124, 126-131. [CrossRef]

3. Khalil, R.; Al Horani, M.; Yousef, A.; Sababheh, M. A new definition of fractional derivative. J. Comput. Appl. Math. 2014, 264, 65-70.

4. Gholami, Y.; Ghanbari, K. New class of conformable derivatives and applications to differential impulsive systems. SeMA J. 2018, 75, 305-333. [CrossRef]

5. Dedovic, N.; Birgani, O.T.; Chandok, S.; Radenovic, S. A note on some recent results of the conformable derivative. Adv. Theory Nonlinear Anal. Its Appl. 2019, 3, 11-17. [CrossRef]

6. Yang, S.; Wang, L.; Zhang, S. Conformable derivative: Application to non-Darcian flow in low-permeability porous media. Appl. Math. Lett. 2018, 79, 105-110.

7. Meng, S.; Cui, Y. The Extremal Solution to Conformable Fractional Differential Equations Involving Integral Boundary Condition. Mathematics 2019, 7, 186. [CrossRef]

8. Kaplan, M.; Akbulut, A. Application of two different algorithms to the approximate long water wave equation with conformable fractional derivative. Arab J. Basic Appl. Sci. 2018, 25, 77-84. [CrossRef] 
9. Usta, F. A conformable calculus of radial basis functions and its applications. Int. J. Optim. Control Theor. Appl. 2018, 8, 178-182. [CrossRef]

10. Foondun, M.; Liu, W.; Tian, K. Moment bounds for a class of Fractional Stochastic Heat Equations. Ann. Probab. 2017, 45, 2131-2153. [CrossRef]

11. Mijena, J.; Nane, E. Space-time fractional stochastic partial differential equations. Stoch. Process. Appl. 2015, 159, 3301-3326. [CrossRef]

12. Omaba, M.E. Some Properties of a Class of Stochastic Heat Equations. Ph.D Thesis, Loughborough University, Loughborough, UK, 2014.

13. Omaba, M.E. On Space-Time Fractional Heat Type Non-Homogeneous Time-Fractional Poisson Equation. J. Adv. Math. Comput. Sci. 2018, 28, 1-18. [CrossRef]

14. Walsh, J.B. An Introduction to Stochastic Partial Differential Equations; Lecture Notes in Maths 1180; Springer: Berlin, Germany, 1986; pp. 265-439.

15. Foondun, M.; Liu, W.; Omaba, M.E. On Some Properties of a class of Fractional Stochastic Heat Equations. J. Theor. Probab. 2017, 30, 1310-1333.

16. Foondun, M.; Nane, E. Asymptotic properties of some space-time fractional stochastic equations. Math. Z. 2017, 287, 493-519. [CrossRef]

17. Omaba, M.E. On Space-Fractional Heat Equation with Non-homogeneous Fractional Time Poisson Process. Progr. Fract. Differ. Appl. 2019, 6, 1-13.

18. Zhong, W.; Wang, L. Basic theory of initial value problems of conformable fractional differential equations. Adv. Differ. Equ. 2018, 2018, 321. [CrossRef]

19. Salahshour, S.; Ahmadian, A.; Ismail, F.; Baleanu, D.; Senu, N. A New fractional derivative for differential equation of fractional order under internal uncertainty. Adv. Mech. Eng. 2015, 7, 1687814015619138. [CrossRef]

20. Al-Refa, M.; Abdeljawad, T. Fundamental Results of conformable Strum-Liouville Eigenvalue Problems. Complexity 2017, 2017, 3720471.

21. Abdeljawad, T. On conformable fractional calculus. J. Comput. Appl. Math. 2015, 279, 57-66. [CrossRef]

22. Sakthivel, R.; Revathi, P.; Ren, Y. Existence of solutions for nonlinear fractional stochastic differential equations. Nonlinear Anal. 2013, 81, 70-86. [CrossRef]

23. Zhang, X.; Agarwal, P.; Liu, Z.; Peng, H.; You, F.; Zhu, Y. Existence and uniqueness of solutions for stochastic differential equations of fractional-order $q>1$ with finite delays. Adv. Differ. Equ. 2017, 2017, 123. [CrossRef]

24. Lakshmikantham, V.; Vatsala, A.S. Basic theory of fractional differential equations. Nonlinear Anal. 2008, 69, 2677-2682. [CrossRef]

25. Iyiola, O.S.; Nwaeze, E.R. Some new results on the new conformable fractional calculus with application using $\mathrm{D}^{\prime}$ Alambert approach. Progr. Fract. Differ. Appl. 2016, 2, 115-121. [CrossRef]

26. Nwaeze, E.R. A Mean Value Theorem for the Conformable fractional calculus on arbitrary time scales. Progr. Fract. Differ. Appl. 2016, 2, 287-291. [CrossRef]

27. Neuman, E. Inequalities and Bounds for the Incomplete Gamma Function. Results Math. 2013, 63, 1209-1214. [CrossRef]

(C) 2019 by the authors. Licensee MDPI, Basel, Switzerland. This article is an open access article distributed under the terms and conditions of the Creative Commons Attribution (CC BY) license (http:/ / creativecommons.org/licenses/by/4.0/). 